\title{
EVOLUCIÓN DE LAS TEMPERATURAS MEDIAS DE LA ESTACIÓN CÁLIDA (ABRIL-SEPTIEMBRE) EN LA MITAD NORTE DE ESPAÑA DURANTE LA PEQUEÑA EDAD DEL HIELO (SIGLOS XVI AL XIX)
}

\author{
Miguel Ángel Saz Sánchez \\ Departamento de Geografía y Ordenación del Territorio \\ Universidad de Zaragoza, Pedro Cerbuna, 12 - 50009 Zaragoza (España) \\ masaz@unizar.es
}

\begin{abstract}
Resumen: En este trabajo se presentan las series reconstruidas mediante técnicas dendroclimáticas de las temperaturas medias del periodo cálido del año (abril-septiembre) durante la Pequeña Edad del Hielo (PEH) en ocho observatorios instrumentales de la mitad norte de España, representativos de distintos ambientes climáticos. Durante este periodo, el deterioro térmico más importante se produce en el siglo XVI, en sus tres primeras décadas y en las de los cincuenta, sesenta y setenta. Los siglos XVII y XVIII se muestran más suaves, detectándose un nuevo descenso de las temperaturas en el XIX. Las crisis frías identificadas coinciden con episodios del mismo signo descritos en estudios realizados en otras zonas de Europa, si bien en la mitad norte de España no se detectan crisis frías importantes en el XVII, centuria señalada por las series hemisféricas por albergar el momento de mayor intensidad de la PEH.
\end{abstract}

Palabras clave: Paleoclimatología, dendroclimatología, Pequeña Edad del Hielo, reconstrucciones de temperaturas, mitad norte de España.

\begin{abstract}
In this work, we present the dendroclimatic reconstruction of the mean temperatures of the warm season (april to September) during the Little Ice Age (LIA) in eight weather stations located in different climatic conditions in the north half of Spain. The most important cold phases appears in 16th century, during the three first decades and in the 50's, 60's and 70's. Temperatures are warmer in 17th and 18th centuries, with a new cold phase in the 19th century. These cold episodes agree with others of the same sign and chronology identified in other European regions, although in the north half of Spain we have not identified important cold phases in the 17 th century, period in which hemispheric mean temperature series indicate the coldest phases into LIA.
\end{abstract}

* Recibido: 06-11-07. Aceptado: 30-11-07. 
Keywords: Paleoclimatology, dendroclimatology, Little Ice Age, temperatures reconstruction, North Half of Spain.

\section{Introducción}

El clima constituye uno de los elementos más notables del paisaje, capaz de condicionar la geomorfología, hidrología, vegetación, fauna e incluso el desarrollo de las actividades humanas y su expresión sobre el territorio. Sin embargo, sus características no son constantes a lo largo del tiempo, variando a distintas escalas temporales que van desde la estacional y anual a la secular y plurisecular.

Antes de la masiva intervención antrópica sobre el sistema climático con la generalización de la industrialización en el siglo XIX, esas variaciones respondían a la evolución normal de un sistema complejo en permanente estado de equilibrio dinámico, en el que cambios en el balance de radiación terrestre provocados a distintas escalas temporales por mecanismos internos y externos al sistema (Goodes et al, 1992), podían alterar las condiciones climáticas a escala global, regional o local. Inserta dentro de esta variabilidad natural del clima estaría la Pequeña Edad del Hielo (PEH), uno de los episodios climáticos más importantes del tramo final del Holoceno. Su comienzo en Europa suele situarse a lo largo del siglo XVI (Briffa et al, 1999, Briffa et al, 2001) aunque otros autores lo localizan en las últimas décadas del XV (Lamb, 1988; Lockwood, 1979). Respecto al final, que como el comienzo no se produce de forma abrupta ni generalizada, parece haber mayor coincidencia entre los investigadores, señalándose las décadas centrales del siglo XIX como el momento en el que cesarían las anomalías térmicas y pluviométricas relacionadas con este episodio (Jones et al, 1998; Mann et al, 1998).

En los últimos años se han multiplicado las investigaciones sobre la evolución del clima durante la PEH. Los resultados obtenidos en distintas regiones del planeta muestran que su dinámica espacial y temporal es más compleja que la que se desprende de su habitual categorización como episodio frío dentro del Holoceno. En este periodo se producen distintas pulsaciones frías, de una duración que oscila entre una y varias décadas y descensos medios de las temperaturas que pueden superar $1^{\circ}$ C, entre las que no es inhabitual encontrar periodos con temperaturas más suaves y promedios similares a los actuales. La secuencia temporal e intensidad de estas anomalías frías o cálidas no es la misma a escala continental y mucho menos hemisférica. Los patrones observados de evolución de las temperaturas durante la PEH en distintos ámbitos geográficos muestran diferencias en ocasiones significativas, detectándose crisis frías que coinciden con condiciones más cálidas en espacios cercanos (Briffa et al, 2004). Las fases frías muestran una mayor coincidencia espacial que las 
cálidas (Jones y Briffa, 2001), aunque la duración y magnitud del enfriamiento difiere entre las zonas afectadas por el deterioro térmico.

La Pequeña Edad del Hielo no se trata así de un periodo en el que se produzca un descenso de las temperaturas continuado en el tiempo y uniforme en el espacio. Para conocer su dinámica interna y tratar de inferir las causas atmosféricas que condicionaron la evolución del clima durante esos siglos, se hace necesario disponer de un mosaico lo más completo posible de reconstrucciones climáticas de carácter regional. Estos estudios indican, a ese nivel territorial, la magnitud, signo y cronología de las principales anomalías, información a partir de la que podremos establecer una pauta evolutiva del clima de las últimas centurias generalizable a nivel hemisférico y que podemos interpretar en términos de Circulación General Atmosférica (CGA).

La Península Ibérica y por extensión todo el extremo meridional de Europa, constituyen espacios de gran interés para el estudio de las variaciones del clima. Por su posición latitudinal, en el tercio sur de la zona de circulación templada ya en contacto con las altas presiones subtropicales, cualquier cambio en el comportamiento habitual de la CGA puede provocar anomalías importantes en los elementos del clima, afectando con severidad las alteraciones de mayor duración a los frágiles ecosistemas mediterráneos. Se trata, por otro lado, de espacios que ofrecen un elevado grado de incertidumbre respecto a su evolución futura en cualquiera de los escenarios previstos por los modelos climáticos. En ese sentido, destaca la importancia que los estudios sobre la evolución del clima en el pasado tienen para validar esos modelos y evaluar la magnitud y trascendencia de las anomalías detectadas en el periodo actual, al poder ser puestas en relación con las que se produjeron en momentos en los que no existía intervención antrópica sobre el sistema climático.

En este trabajo se presenta la evolución de las temperaturas medias del semestre cálido del año (desde abril a septiembre) entre los siglos XVI y XIX en ocho puntos de la mitad septentrional de España. Se trata de reconstrucciones realizadas mediante técnicas dendroclimáticas sobre los datos de ocho observatorios instrumentales representativos de ambientes climáticos diferenciados. En ellos, las distintas influencias que sobre los patrones generales que rigen la CGA en las latitudes templadas ejercen el Océano Atlántico y el Mar Mediterráneo, las masas de aire euroasiática y africana o la configuración del relieve, hacen que las variables climáticas muestren diferencias espaciales marcadas.

Esta información reconstruida, además de contribuir al conocimiento de la evolución del clima europeo de las últimas centurias, nos permitirá conocer la cronología y magnitud de las principales anomalías térmicas registradas en la mitad norte de España durante la Pequeña Edad del Hielo y su relación con los patrones de evolución de las temperaturas identificados en otras zonas de Europa. 


\section{Las reconstrucciones dendroclimáticas}

\subsection{Antecedentes}

A lo largo de la segunda mitad de la década de los años ochenta pero fundamentalmente a lo largo de la de los noventa, se desarrolló en España un amplio banco de información dendrocronológica, fundamental para cualquier intento de reconstrucción del clima mediante técnicas basadas en la dendroclimatología. En este punto ha de hacerse una especial mención a los continuados trabajos de campo y laboratorio emprendidos por los doctores José Creus Novau (CSIC), Ángel Fernández Cancio (INIA) y Mar Génova Fuster (EUITF) para crear este banco de cronologías, con el soporte de varios proyectos de investigación financiados por organismos nacionales e internacionales (podemos señalar entre los más importantes: Comisión Interministerial de Ciencia y Tecnología CICYT: FOR 89-0866-C02-01; Comunidad Europea: FI2W-CT91-0075; CICYT: CLI96-1862; Ministerio de Agricultura: FO96-013; Comunidad Europea: Contract ENVA-CT97-0641, además de otros financiados por el ICONA, entre 1988 y 1990, y la Xunta de Galicia, entre 1991 y 1993).

En la actualidad, este banco dendrocronológico está compuesto por algo más de 50 cronologías, que agrupan muestras del crecimiento radial de más de 1.500 árboles, siendo las coníferas el grupo que supone un porcentaje más elevado, superior al $90 \%$. Con esa base de datos dendrocronológicos ya se han realizado en España algunas reconstrucciones de distintas variables climáticas, cuyos resultados pueden consultarse entre otros en Creus y Puigdefábregas (1983), Creus et al (1995), Creus et al (1996), Fernández et al (1996), Creus y Saz (1999), Manrique y Fernández-Cancio (1999), Candela (2000), Manrique y Fernández-Cancio (2000), Saz y Creus (2000), Saz y Creus (2001a), Saz y Creus (2001b), Saz, (2003), Saz y Creus (2003), Saz et al, (2003), Saz et al 2004, Saz et al 2005, Saz y Creus 2005, Saz y Creus, 2004; Saz, 2005.

\subsection{Construcción de las cronologías}

Para la construcción de estas cronologías (fig. 1) se siguió la metodología dendrocronológica tradicional (Fritts, 1990, 1991; Cook y Kairiukstis, 1990; Guiot, 1990).

En las zonas muestreadas se seleccionaron entre 20 y 25 árboles vivos, de los que se extrajeron las muestras de su crecimiento radial (cores). El criterio de selección de los árboles no es aleatorio, sino que se eligen ejemplares situados en zonas con un 
elevado estrés climático (cerca de sus límites latitudinales o altitudinales de distribución, sobre suelos poco desarrollados, libres de competencia, etc) y con un aspecto exterior (forma de la copa, rugosidad del tronco y diámetro) que indique una elevada longevidad.

Los cores se dejan secar en el laboratorio a temperatura ambiente durante un periodo de entre 7 y 14 días, colocándose posteriormente en soportes de madera para evitar su deterioro. Después, se cortan longitudinalmente y se pulen con lijas de grano sucesivamente más fino hasta obtener una buena visión de la secuencia de anillos de crecimiento radial.

La anchura de los anillos se midió en un sistema tipo ANIOL, con una precisión de $0,01 \mathrm{~mm}$, que permite la digitalización automática de las mediciones por medio del programa CATRAS (Aniol, 1983). Para obtener una datación correcta de cada uno de los anillos, las series de mediciones son testadas con el programa COFECHA (Holmes, 1997), que mediante la comparación estadística de las mediciones efectuadas sobre cada muestra con una serie maestra, nos indica aquellos segmentos en los

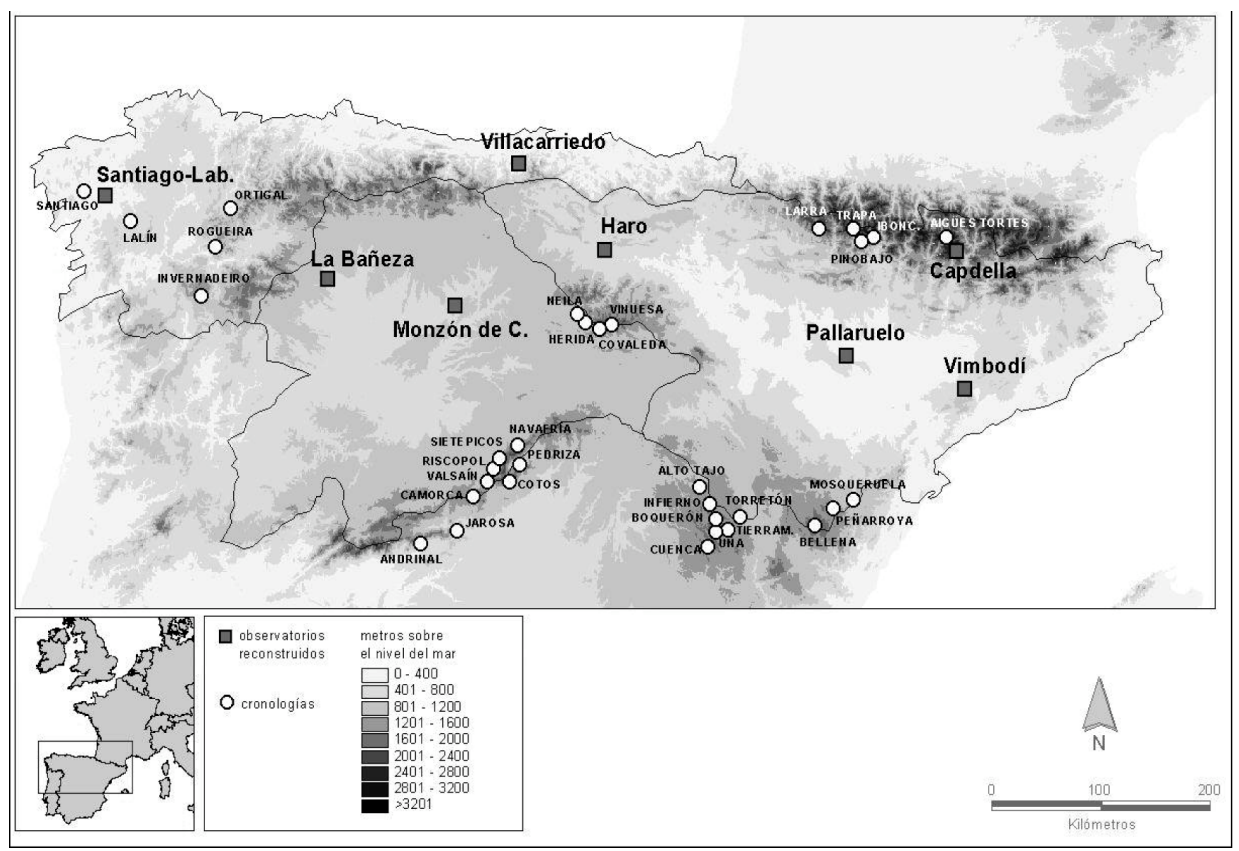

Figura 1. Localización de las cronologías y de los observatorios reconstruidos. 
que hay problemas de sincronización, indicando el número de anillos que hay que insertar o eliminar de la serie para mejorar su sincronización con el resto.

Una vez sincronizadas, las series de crecimiento han de ser estandarizadas con objeto de eliminar la tendencia que cada una de ellas muestra en función de la edad del árbol. Para ello se utilizó el programa ARSTAN, que ajusta cada serie a un modelo teórico de crecimiento, generalmente una función exponencial decreciente o una spline de distinta amplitud, en torno al que fluctúan los valores reales de cada año. Estos valores se transforman en índices relacionando el crecimiento real con el teórico del modelo, obteniéndose series en las que se ha estabilizado la media y homogeneizado la varianza. Finalmente se utilizó la cronología estándar por aportar una señal climática más fuerte y mejorar la señal común reconstruible. El conjunto de muestras de una localidad, sincronizadas estandarizadas y transformadas en índices, pueden agruparse para formar una cronología local, independientemente de la edad de cada árbol.

\subsection{Reconstrucción de las variables climáticas}

En el proceso de reconstrucción de las variables climáticas se aplicó la metodología propuesta por Manrique (1997) y Fernández y Manrique (1997), basada en una modificación del método tradicional de reconstrucción aplicado en este tipo de estudios (Fritts, 1976; Cook y Kairiukstis, 1990). La nueva propuesta metodológica se basa en la existencia de importantes relaciones entre elementos del clima registrados en observatorios situados a distancias espaciales importantes (Villa et al, 1985). Esta característica es extrapolable también a las series dendroclimáticas, como lo demuestra la sincronización existente entre muestras obtenidas de árboles distantes entre sí más de $400 \mathrm{Km}$ (Génova, 1988), lo que permitiría la utilización conjunta de un mayor número de cronologías para reconstruir el clima de un lugar determinado (Fernández y Manrique, 1997), aunque las muestras de los árboles seleccionados procedan de zonas alejadas del observatorio instrumental con el que se calibra la información. El uso de más cronologías, que funcionan como predictores en el proceso de reconstrucción, servirá para reconstruir un número mayor de variables climáticas, así como para prolongar en el tiempo esas reconstrucciones.

Bajo estos nuevos planteamientos metodológicos, las series de crecimiento que forman las cronologías y que, por tanto, intervienen en el proceso de reconstrucción, se seleccionan en función de su correlación con las variables climáticas. De esta forma se configuran regiones dendrocronológicas, con criterios de coherencia geo- 
gráfica y uniformidad climáticas. Las series de crecimiento de cada una de esas regiones se sincronizan con el programa COFECHA y posteriormente se estandarizan con ARSTAN, obteniéndose finalmente una cronología estándar de la zona.

Los observatorios que se seleccionaron para la reconstrucción de los valores preinstrumentales de temperatura son, como ya se ha señalado, representativos de ambientes climáticos diferenciados dentro de la mitad septentrional de España: desde zonas con caracteres oceánicos a otras plenamente mediterráneas pasando por espacios con matices de continentalidad más o menos acusados y otros situados en áreas de montaña. Además de este criterio de carácter geográfico, su selección se realizó teniendo en cuenta la longitud de sus series de registros instrumentales (al menos 40 años) y su calidad (escaso porcentaje de lagunas y ausencia de inhomogeneidades significativas). Las lagunas se rellenaron mediante modelos de regresión simple con las series de observatorios vecinos, mientras que la homogeneidad se evaluó con el Standard Normal Homogeneity Test (Alexandersson, 1986), no detectándose en las series instrumentales de temperatura de los observatorios elegidos inhomogeneidades significativas.

Para la reconstrucción (una descripción más detallada puede consultarse en Fernández y Manrique, 1997) se calculó la regresión de los componentes principales entre las variables climáticas y las series de crecimientos considerados para los años $t$ y $t$-1. La fiabilidad del resultado se analiza a través de los coeficientes de correlación del proceso de calibración-verificación aplicado a distintos intervalos mediante técnicas bootstrap (programa PRECON-K) y calculando el número de series de anillos que por azar tendrían correlación significativa con las variables climáticas. Esta nueva variable se compara con algunas distribuciones de probabilidad (normal, weibull o gamma, entre otras) para conocer si las reconstrucciones son distintas de un simple

Tabla 1. Varianza explicada $\left(R^{2}\right)$ por el modelo construido para explicar el crecimiento en función del clima (función respuesta).

\begin{tabular}{lcc}
\hline Observatorio & $\left(R^{2}\right)$ segundo trimestre & $\left(R^{2}\right)$ tercer trimestre \\
\hline Haro & 0,65 & 0,61 \\
Pallaruelo & 0,34 & 0,68 \\
Capdella & 0,75 & 0,80 \\
Vimbodí & 0,69 & 0,70 \\
La Bañeza & 0,41 & 0,62 \\
Monzón de Campos & 0,69 & 0,64 \\
Santiago-Labacolla & 0,57 & 0,48 \\
Villacarriedo & 0,72 & 0,69 \\
\hline
\end{tabular}


proceso aleatorio. Por tanto, una variable climática reconstruida se considera significativa si supera los procesos de calibración, verificación y simulación.

El proceso metodológico señalado y el conjunto de cronologías disponibles, permitieron la reconstrucción de las temperaturas medias del segundo y tercer trimestres (abril, mayo y junio; julio, agosto y septiembre), que fueron agrupadas para su análisis temporal posterior como estación cálida (abril-septiembre), de ocho observatorios de la mitad norte de España: cuatro localizados en la Cuenca del Ebro: Haro

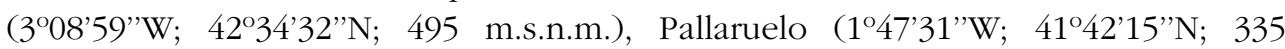

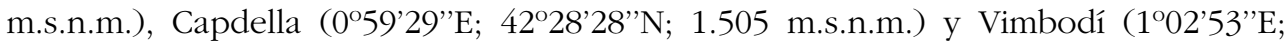
$41^{\circ} 23^{\prime} 55^{\prime \prime} \mathrm{N}$; 490 m.s.n.m.); dos en el Valle del Duero: Monzón de Campos (5³0'24”W;

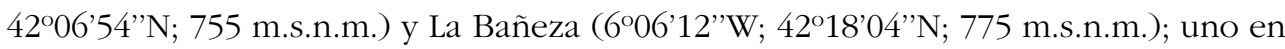

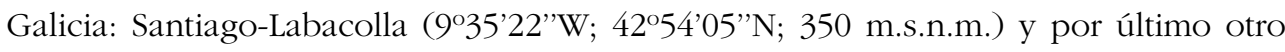

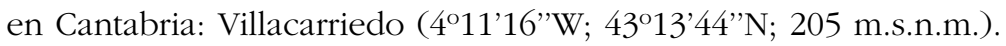

Todas las reconstrucciones, excepto la realizada en Monzón de Campos (que arranca en el año 1604), muestran la evolución de las temperaturas desde comienzos del siglo XVI. Su fiabilidad viene determinada por la proporción de varianza explicada por el modelo construido para explicar el crecimiento en función del clima (función respuesta). En la tabla 1 se señalan los valores obtenidos en las ocho reconstrucciones, que en todos los casos superan ampliamente el valor citado como significativo $(0,32)$ en la bibliografía.

\section{Los datos reconstruidos. Análisis climático desde una perspectiva plurisecular}

Los datos reconstruidos han sido representados gráficamente en la figura 2. Para destacar las oscilaciones frías o cálidas de amplitud temporal decenal o pluridecenal, más allá de la presencia de años o grupos de años de signo contrario, se ha representado, además, el suavizado de los datos mediante filtro gaussiano de paso bajo y una longitud de 10 años.

A la vista de esas gráficas, cabría en primer lugar destacar en las reconstrucciones realizadas en la mitad norte de España la ausencia de un enfriamiento continuado durante el periodo en el que se inscribe la Pequeña Edad del Hielo, apareciendo oscilaciones frías importantes en todos los observatorios junto a periodos de temperaturas más suaves. Este aspecto es característico de la PEH en cualquier área geográfica, 


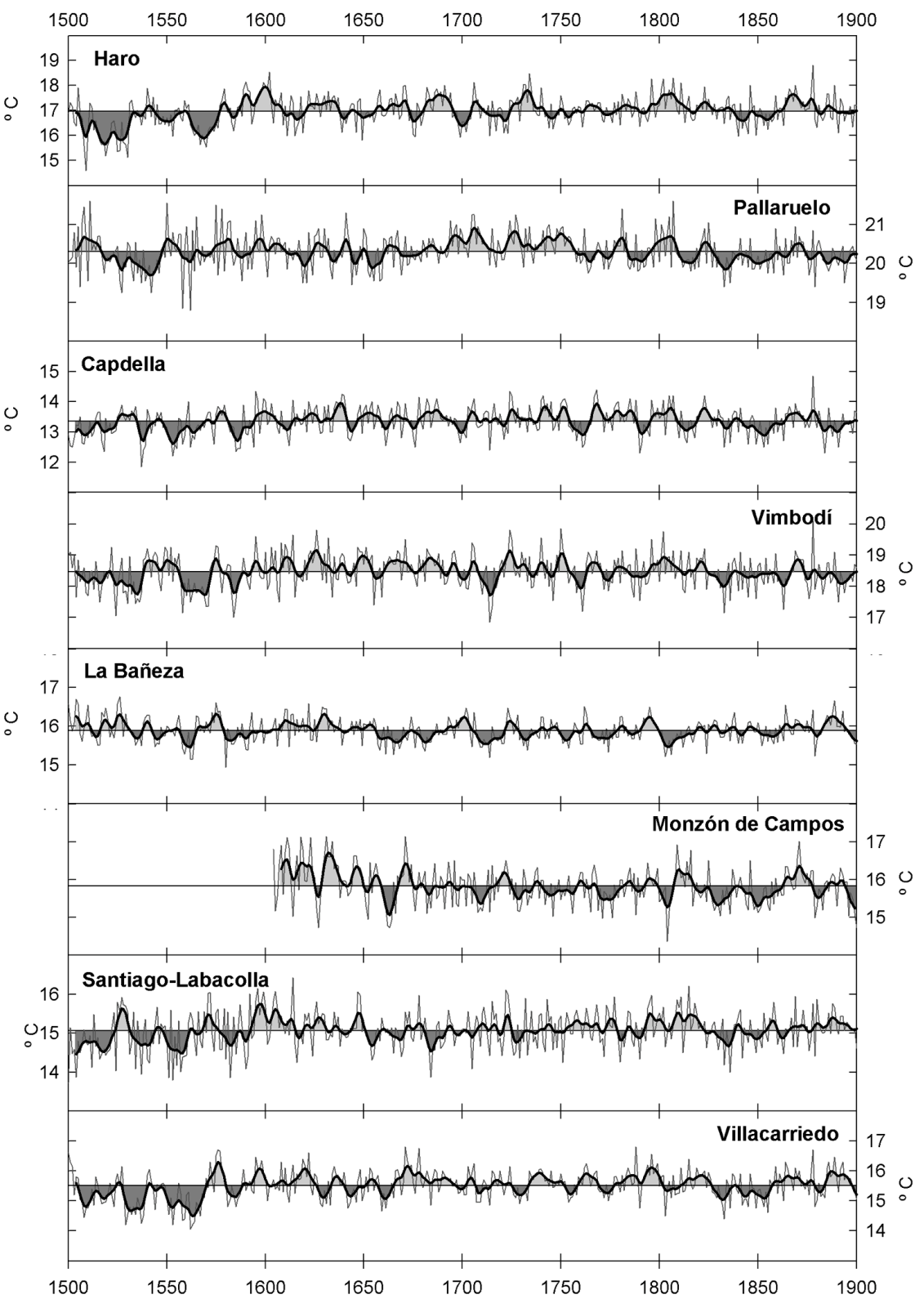

Figura 2. Valores de la temperatura media de la estación cálida (abril-septiembre) en los observatorios reconstruidos en la mitad norte de España entre 1500 y 1900. La curva negra representa un suavizado de los datos mediante filtro gaussiano de paso bajo y longitud de 10 años. La línea negra señala la temperatura media del periodo reconstruido. 
habiendo sido señalado por Lamb (1977) en sus trabajos sobre la evolución del clima europeo en las últimas centurias a partir de fuentes históricas y subrayado por, entre otros, Bradley y Jones (1993), Mann et al (1998), Luckmann (2000) y Nesje y Dahl (2003) mediante la información obtenida con distintas fuentes de proxy-data.

Para situar en el tiempo los eventos fríos de mayor magnitud y los periodos de temperaturas más suaves, en suma las oscilaciones de media y alta frecuencia que jalonarían la evolución de las temperaturas en el área de estudio durante la PEH, se han señalado en las tablas 2 y 3 aquellos periodos de 50, 25 y 10 años con una temperatura media reconstruida más baja y más elevada, así como la diferencia, negativa o positiva, en grados Celsius respecto de la media del periodo 1500-1900. A continuación, pasamos a describir los aspectos más relevantes de la evolución de las temperaturas del semestre abril-septiembre en la mitad norte de España desde el siglo XVI.

\subsection{Las crisis frías del siglo XVI}

El siglo XVI constituye uno de los periodos en los que la temperatura media del semestre abril-septiembre ofrece los valores más bajos a lo largo de los cuatro siglos en los que disponemos de información. Los valores reconstruidos en esta centuria en Haro, Pallaruelo, Capdella, Vimbodí, Santiago-Labacolla y Villacarriedo (recordemos que en Monzón de Campos no hay datos para el siglo XVI) quedan en su mayoría por debajo de la media del conjunto de los cuatro siglos analizados, localizándose además en este siglo en todos los observatorios (excepto La Bañeza) el periodo de cincuenta años con una media más baja, con diferencias respecto al promedio del periodo 1500-1900 que, como señalamos en la tabla 2, oscilan según los datos reconstruidos entre los $0,2^{\circ} \mathrm{C}$ y $\operatorname{los} 0,5^{\circ} \mathrm{C}$. En periodos de 25 años (en todos los observatorios los periodos de esta longitud más fríos aparecen en el siglo XVI) las diferencias pueden superar $1^{\circ} \mathrm{C}$, como es el caso de Haro en el 1506-1530 y en el 1517-1526, aunque en general las diferencias son más moderadas, oscilando en el resto de los observatorios entre $\operatorname{los} 0,3{ }^{\circ} \mathrm{C}$ y los $0,5^{\circ} \mathrm{C}$ en periodos de 25 años y entre los $0,4^{\circ} \mathrm{C}$ y los $0,9^{\circ} \mathrm{C}$ en periodos de 10 años.

Es seguramente en las tres primeras décadas del siglo cuando se observa la que se presenta como la crisis fría más generalizada de las temperaturas de época cálida del año en la mitad norte de España y en conjunto de mayor magnitud en el contexto de la PEH. Al señalado descenso térmico de $1{ }^{\circ} \mathrm{C}$ en Haro durante el periodo 1506- 
Tabla 2. Periodos de 10, 25 y 50 años más fríos en las series reconstruidas de la temperatura media de la estación cálida (abr-sept) en la mitad norte de España.

\begin{tabular}{|c|c|c|c|}
\hline \multicolumn{4}{|c|}{ PERIODOS DE DIEZ AÑOS MÁS FRÍOS } \\
\hline Haro & Pallaruelo & Capdella & Vimbodi \\
\hline $1517-1526(-1,2)$ & $1537-1546(-0,5)$ & $1550-1559(-0,5)$ & $1562-1571(-0,7)$ \\
\hline $1564-1573(-0,9)$ & $1526-1535(-0,4)$ & $1501-1510(-0,5)$ & $1528-1537(-0,6)$ \\
\hline $1507-1516(-0,8)$ & $1827-1836(-0,4)$ & $1585-1594(-0,4)$ & $1709-1718(-0,5)$ \\
\hline $1544-1553(-0,4)$ & $1518-1527(-0,3)$ & $1849-1858(-0,4)$ & $1583-1592(-0,4)$ \\
\hline $1695-1704(-0,4)$ & $1652-1661(-0,3)$ & $1754-1763(-0,3)$ & $1509-1518(-0,3)$ \\
\hline La Bañeza & Monzón de $C$. & Santiago-Lab. & Villacarriedo \\
\hline $1555-1564(-0,3)$ & $1827-1836(-0,5)$ & $1551-1560(-0,6)$ & $1558-1567(-0,9)$ \\
\hline $1709-1718(-0,3)$ & $1847-1856(-0,4)$ & $1500-1509(-0,5)$ & $1533-1537(-0,9)$ \\
\hline $1803-1812(-0,3)$ & $1658-1667(-0,4)$ & $1512-1521(-0,4)$ & $1713-1717(-0,8)$ \\
\hline $1661-1670(-0,3)$ & $1769-1778(-0,4)$ & $1534-1543(-0,4)$ & $1558-1562(-0,7)$ \\
\hline $1582-1591(-0,2)$ & $1705-1714(-0,3)$ & $1682-1691(-0,4)$ & $1583-1587(-0,7)$ \\
\hline \multicolumn{4}{|c|}{ PERIODOS DE VEINTICINCO AÑOS MÁS FRÍOS } \\
\hline Haro & Pallaruelo & Capdella & Vimbodi \\
\hline $1506-1530(-1,0)$ & $1522-1546(-0,4)$ & $1500-1524(-0,3)$ & $1513-1537(-0,4)$ \\
\hline $1551-1575(-0,5)$ & $1831-1855(-0,3)$ & $1536-1560(-0,3)$ & $1562-1586(-0,4)$ \\
\hline $1839-1863(-0,2)$ & $1635-1659(-0,2)$ & $1823-1857(-0,2)$ & $1825-1849(-0,2)$ \\
\hline La Bañeza & Monzón de C. & Santiago-Lab. & Villacarriedo \\
\hline $1506-1530(-0,2)$ & $1828-1852(-0,3)$ & $1500-1524(-0,4)$ & $1544-1568(-0,6)$ \\
\hline $1800-1824(-0,2)$ & $1769-1793(-0,2)$ & $1536-1560(-0,3)$ & $1515-1539(-0,5)$ \\
\hline $1709-1733(-0,1)$ & $1728-1752(-0,2)$ & $1682-1706(-0,2)$ & $1831-1855(-0,3)$ \\
\hline \multicolumn{4}{|c|}{ PERIODOS DE CINCUENTA AÑOS MÁS FRÍOS } \\
\hline Haro & Pallaruelo & Capdella & Vimbodi \\
\hline $1507-1556(-0,5)$ & $1518-1567(-0,2)$ & $1537-1586(-0,2)$ & $1523-1572(-0,3)$ \\
\hline $1816-1865(-0,1)$ & $1809-1858(-0,2)$ & $1809-1858(-0,1)$ & $1828-1877(-0,1)$ \\
\hline La Bañeza & Monzón de C. & Santiago-Lab. & Villacarriedo \\
\hline $1707-1756(-0,1)$ & $1817-1866(-0,2)$ & $1511-1560(-0,2)$ & $1518-1567(-0,5)$ \\
\hline $1639-1688(-0,1)$ & $1728-1777(-0,1)$ & $1651-1700(-0,1)$ & $1806-1855(-0,1)$ \\
\hline
\end{tabular}

1530 se suman los detectados en Pallaruelo en el intervalo 1522-1546, en Capdella en el 1500-1524, en Vimbodí en el 1513-1537, en La Bañeza en el 1506-1530 y en Santiago-Labacolla en el 1500-1524, en todos los casos los periodos de 25 años con una temperatura media del semestre abril-septiembre más baja a lo largo de los cuatro siglos reconstruidos, con diferencias que según la información reconstruida son de entre $0,2^{\circ} \mathrm{C}$ y $0,5^{\circ} \mathrm{C}$, mientras que en Villacarriedo el $1515-1539$ aparece también 
Tabla 3. Periodos de 10, 25 y 50 años más cálidos en las series reconstruidas de la temperatura media de la estación cálida (abr-sept) en la mitad norte de España.

\begin{tabular}{|c|c|c|c|}
\hline \multicolumn{4}{|c|}{ PERIODOS DE DIEZ AÑOS MÁS CÁLIDOS } \\
\hline Haro & Pallaruelo & Capdella & Vimbodi \\
\hline $1594-1603(+0,7)$ & $1704-1713(+0,4)$ & $1632-1641(+0,4)$ & $1623-1632(+0,5)$ \\
\hline $1800-1809(+0,6)$ & $1798-1807(+0,4)$ & $1595-1604(+0,4)$ & $1723-1732(+0,4)$ \\
\hline $1682-1691(+0,6)$ & $1743-1752(+0,4)$ & $1798-1807(+0,4)$ & $1796-1805(+0,4)$ \\
\hline $1726-1735(+0,6)$ & $1723-1732(+0,4)$ & $1777-1786(+0,4)$ & $1645-1654(+0,4)$ \\
\hline $1864-1873(+0,6)$ & $1507-1516(+0,4)$ & $1681-1690(+0,3)$ & $1662-1671(+0,4)$ \\
\hline La Bañeza & Monzón de C. & Santiago-Lab. & Villacarriedo \\
\hline $1884-1893(+0,3)$ & $1629-1638(+0,6)$ & $1596-1605(+0,5)$ & $1670-1679(+0,5)$ \\
\hline $1517-1526(+0,3)$ & $1610-1619(+0,6)$ & $1809-1818(+0,4)$ & $1570-1579(+0,5)$ \\
\hline $1570-1579(+0,3)$ & $1807-1816(+0,4)$ & $1794-1803(+0,3)$ & $1793-1802(+0,5)$ \\
\hline $1627-1636(+0,3)$ & $1864-1873(+0,4)$ & $1880-1889(+0,3)$ & $1885-1894(+0,4)$ \\
\hline $1501-1510(+0,3)$ & $1665-1674(+0,3)$ & $1715-1724(+0,3)$ & $1613-1622(+0,4)$ \\
\hline \multicolumn{4}{|c|}{ PERIODOS DE VEINTICINCO AÑOS MÁS CÁLIDOS } \\
\hline Haro & Pallaruelo & Capdella & Vimbodi \\
\hline $1586-1660(+0,5)$ & $1692-1716(+0,3)$ & $1765-1789(+0,3)$ & $1608-1632(+0,3)$ \\
\hline $1801-1825(+0,4)$ & $1727-1751(+0,3)$ & $1617-1641(+0,2)$ & $1647-1671(+0,3)$ \\
\hline $1865-1889(+0,3)$ & $1575-1599(+0,2)$ & $1724-1748(+0,2)$ & $1719-1743(+0,2)$ \\
\hline La Bañeza & Monzón de C. & Santiago-Lab. & Villacarriedo \\
\hline $1610-1634(+0,5)$ & $1610-1634(+0,5)$ & $1591-1615(+0,3)$ & $1667-1691(+0,3)$ \\
\hline $1503-1527(+0,2)$ & $1635-1659(+0,2)$ & $1794-1818(+0,3)$ & $1778-1802(+0,3)$ \\
\hline $1869-1893(+0,2)$ & 1864-1888 $(+0,1)$ & $1753-1777(+0,2)$ & $1598-1622(+0,2)$ \\
\hline \multicolumn{4}{|c|}{ PERIODOS DE CINCUENTA AÑOS MÁS CÁLIDOS } \\
\hline Haro & Pallaruelo & Capdella & Vimbodi \\
\hline $1589-1638(+0,3)$ & $1704-1753(+0,3)$ & $1740-1789(+0,2)$ & $1622-1671(+0,2)$ \\
\hline $1777-1826(+0,3)$ & $1563-1612(+0,1)$ & $1592-1641(+0,1)$ & $1720-1769(+0,1)$ \\
\hline La Bañeza & Monzón de C. & Santiago-Lab. & Villacarriedo \\
\hline $1607-1656(+0,1)$ & $1607-1656(+0,3)$ & $1592-1641(+0,2)$ & $1777-1826(+0,2)$ \\
\hline $1500-1549(+0,1)$ & $1777-1826(+0,0)$ & $1773-1822(+0,2)$ & $1573-1622(+0,2)$ \\
\hline
\end{tabular}

entre los tres más fríos. Destacarían posteriormente, en todos los puntos con información reconstruida, otras crisis frías importante en las décadas de los cincuenta, sesenta y setenta, como en el periodo 1551-1575 en Haro, el 1536-1560 en Capdella, 1562-1586 en Vimbodí, 1555-1564 en La Bañeza, 1536-1560 en Santiago-Labacolla y 1544-1568 en Villacarriedo. 


\subsection{La recuperación térmica de los siglos XVII y XVIII}

Durante los siglos XVII y XVIII no se detectan sobre las temperaturas medias del semestre analizado crisis frías de la frecuencia, magnitud y generalización espacial de las descritas para el siglo XVI. Aparecen algunos periodos fríos, señalados en la tabla 2, como en Pallaruelo el 1635-1659, en La Bañeza el 1709-1733, en Monzón de Campos el 1769-1793 o en Santiago-Labacolla el 1682-1704, pero son más importantes en estas dos centurias aquellos intervalos con temperaturas medias por encima del umbral que supone la media del periodo 1500-1900.

Podríamos señalar, en primer lugar, el intervalo de temperaturas más suaves que en buena parte de los observatorios se detecta en las primeras décadas del XVII y que, en algunos casos, comienza ya en el tránsito secular. Esta recuperación térmica frente a las crisis frías que caracterizaron el XVI, aparece bien representada en Haro en el periodo 1589-1638, con un incremento medio de $0,3{ }^{\circ} \mathrm{C}$, en Capdella en el 1592 1641, en La Bañeza y Monzón de Campos en el 1607-1656, en Santiago-Labacolla en el 1592-1641 y en Villacarriedo en el 1573-1622. Se trata, en todos los casos, de uno de los dos periodos de 50 años con una temperatura media del semestre cálido más elevada (tabla 3). La identificación, también en estas primeras décadas del siglo XVII, en todos los observatorios reconstruidos de alguno de los periodos de 25 y 10 años con una temperatura media más elevada, subrayaría la presencia de este periodo más cálido en el contexto temporal analizado. Ejemplos serían el intervalo 1594-1603 en Haro, el 1617-1641 en Capdella, el 1618-1632 en Vimbodí, el 1610-1634 en La Bañeza y Monzón de Campos, el 1591-1615 en Santiago-Labacolla y el 1598-1622 en Villacarriedo.

Respecto al siglo XVIII habría de destacarse, primero, el incremento térmico que en el segundo cuarto de la centuria se detecta en los cuatro observatorios reconstruidos en la Cuenca del Ebro, con incrementos medios de $0,6{ }^{\circ} \mathrm{C}$ en Haro entre 1726 y 1735 , de $0,3{ }^{\circ} \mathrm{C}$ en Pallaruelo entre 1727 y $1751,0,2{ }^{\circ} \mathrm{C}$ en Capdella en el 1724-1738 y de $0,2{ }^{\circ} \mathrm{C}$ en Vimbodí en el 1719-1743, y segundo el nuevo periodo de temperaturas más suaves que se detecta en el tramo final de la centuria y en los primeros años del siglo XIX en Haro, Santiago-Labacolla y Villacarriedo.

\subsection{El nuevo deterioro térmico del siglo XIX}

Tras este periodo de dos siglos de temperaturas más suaves, que culmina con ese intervalo cálido señalado para el tránsito secular, el siglo XIX alberga de nuevo temperaturas medias del semestre abril-septiembre bajas en el contexto temporal anali- 
zado. En seis de los ocho observatorios reconstruidos, uno de los dos periodos de 50 años con una media más baja se sitúa en esta centuria (tabla 2): en Haro el 1816-1855, en Pallaruelo y Capdella el 1809-1858, en Vimbodí el 1828-1877, en Monzón de Campos el 1817-1866 y en Villacarriedo el 1806-1855. Esta crisis fría parece adquirir mayor intensidad en el tercio central de la centuria, como indicaría la presencia en esas décadas en Haro (1839-1863), Pallaruelo (1831-1855), Capdella (1823-1857), Vimbodí (1825-1849), Monzón de Campos (1828-1852) y Villacarriedo (1831-1855) de uno de los tres periodos con una media reconstruida más baja, con diferencias de hasta $0,5^{\circ} \mathrm{C}$

\section{Discusión: interpretación de los resultados en el contexto de la evolución observada en otros trabajos de ámbito regional y hemisférico}

Las crisis frías y los periodos más suaves señalados en el apartado anterior, que jalonarían la evolución de las temperaturas medias del semestre cálido (abril-septiembre) en la mitad norte de España durante al Pequeña Edad del Hielo, no resultan acontecimientos aislados en el ámbito regional en el que nos situamos. Su intensidad e incluso su precisión cronológica, muestran algunas diferencias en el conjunto del área de estudio. Sin embargo, la información paleoclimática obtenida en distintas zonas de Europa mediante el uso de proxy-data indicaría, como veremos en este punto, su coincidencia con anomalías del mismo signo en otras zonas del continente, aunque con diferencias que entendemos pueden también resultar significativas.

La evolución de las temperaturas durante el siglo XVI es particularmente interesante, tanto a escala local como continental y hemisférica. Saz y Creus (1999) y Manrique y Fernández (2000) señalan mediante el análisis de datos dendroclimáticos, que uno de los aspectos más característicos del clima de esta centuria en España es la existencia de un elevado número de registros extremos, señalando Heino et al (1999) este mismo aspecto para el clima del centro y norte de Europa tras el examen de distintas fuentes de información proxy.

Las características anómalas del clima en este siglo, relacionadas con esta elevada presencia de extremos y también con la ocurrencia de crisis frías importantes de una duración de entre una y varias décadas, fueron percibidas como algo extraordinario por los contemporáneos, y aunque es a menudo discutible el establecimiento de relaciones causales entre las alteraciones en el clima y determinados acontecimientos his- 
tóricos (De Vries, 1981), lo cierto es que algunos trabajos han destacado la relación de las fluctuaciones climáticas del siglo XVI sobre aspectos como el precio del cereal en Europa (Bauernfeind y Woitek, 1999), la producción vitícola (Landsteiner, 1999), los movimientos naturales de población (Galloway, 1994) e incluso las mentalidades (Behringer, 1999).

En la mitad norte de España hemos señalado la presencia de un deterioro térmico importante de las temperaturas de la estación cálida en las tres primeras décadas del siglo y después, aunque de una magnitud inferior, un nuevo descenso térmico en las de los cincuenta, sesenta y setenta. La presencia de estas dos crisis frías ha sido señalada por otros estudios realizados en el continente euroasiático. Briffa (1995) en Siberia a partir de información dendroclimática, así como Glasser et al (1999) y Pfister y Brázdil (1999) en distintas zonas de centroeuropa a partir de sus trabajos sobre documentación histórica, muestran evidencias de un deterioro de las temperaturas en las primeras décadas de siglo, de menor magnitud en cualquier caso que las crisis frías posteriores. Para la crisis fría de los cincuenta, sesenta y setenta, las referencias son más abundantes en la bibliografía. Briffa et al (1992, 1995) señalan a partir de reconstrucciones dendroclimáticas su presencia en series de paleotemperaturas obtenidas en Fenoscandia. Kalela-Brundin (1999) en el norte de Europa, Hughes et al (1999) en Siberia y Serré-Bachet (1994) en el noreste de Italia, obtuvieron también a partir de reconstrucciones dendroclimáticas evidencias de este deterioro de las temperaturas, mientras que Tarussov (1992) con cores de hielo, Glasser y Hagedorn (1991), Guiot (1992), Grove y Conterio (1998), Koslovsky y Glasser (1999) y Pfister y Brazdil (1999) con documentación histórica, señalan su presencia en distintas zonas del continente europeo.

En los siglos XVII y XVIII no se han detectado, en las series térmicas reconstruidas en este trabajo, crisis frías de la magnitud de las descritas en el XVI, centuria que, según se desprende de estos datos, albergaría el momento de mayor deterioro de las temperaturas medias entre abril y septiembre, en el contexto de la Pequeña Edad del Hielo en la mitad norte de España.

La presencia de temperaturas más suaves durante el siglo XVIII no resulta tampoco desconocida en el ámbito de las investigaciones paleoclimáticas desarrolladas en Europa. Briffa et al (1990, 1992, 1998), Pfister (1992) y Kalela-Brundin (1999) en el centro y norte del continente, así como Serré-Bachet (1994) en el sur, señalan para esta centuria temperaturas más suaves, respecto al siglo XVI pero también frente al XVII. Es precisamente en este siglo XVII, cuando las temperaturas parecen alcanzar sus niveles más bajos en el contexto de la PEH, como señalan Briffa et al (1998), Jones et al (1998), Crowley (2000), Esper et al (2002) y Mann y Jones (2003) a partir 
de las series hemisféricas de paleotemperaturas obtenidas tras integrar la información de una o varias fuentes proxy de distintas zonas el hemisferio norte. En especial durante el Mínimo Maunder (1645-1715), periodo en el que el enfriamiento fue especialmente intenso. Trabajos anteriores realizados con información dendroclimática española (Saz, 2003), indican también la presencia de crisis frías importantes durante el Mínimo Maunder, pero que afectaron especialmente a las temperaturas del primer y cuarto trimestres (ene-feb-mar y oct-nov-dic), aunque sin ser de la magnitud y generalización espacial que mostraron los momentos más fríos del siglo XVI.

La falta de sincronía que muestran las crisis frías más importantes en la PEH entre nuestro área de estudio y lo señalado por las series hemisféricas no es algo inhabitual al comparar las reconstrucciones realizadas en distintos ámbitos geográficos. A diferencia de lo que señalan las series hemisféricas antes citadas, Briffa y Schweingruber (1992) y Kalela-Brundin (1994) en Fenoscandia, así como Serré-Bachet (1994) en el noreste de Italia, indican condiciones estivales más cálidas en el siglo XVII que en la centuria anterior, mientras que D'Arrigo y Jacoby (1992) en Norteamérica y Kameda et al (1992) en Groenlandia, señalan que el mayor enfriamiento durante la PEH en esas áreas geográficas hay que buscarlo en las décadas centrales del siglo XIX. Es precisamente entonces cuando, coincidiendo con el deterioro térmico que se ha señalado en nuestra zona de estudio y con crisis frías identificadas en otras zonas de Europa (Pfister,1992; Serré-Bachet,1994; Koslowsky y Glasser,1999; Kalela-Brundin,1999), otros autores como Jones et al (1998) y Mann et al (1998) indican el final de las anomalías climáticas relacionadas con la PEH.

\section{Conclusiones}

Las series reconstruidas, mediante técnicas dendroclimáticas, de las temperaturas medias de la estación cálida (desde junio a septiembre) entre los siglos XVI y XIX en distintos puntos de la mitad norte de España, muestran la existencia de un deterioro térmico importante durante el siglo XVI. En especial en sus tres primeras décadas, en las que tiene lugar el mayor enfriamiento en el contexto de la Pequeña Edad del Hielo, y en las de los años cincuenta, sesenta y setenta. El tercio central del siglo XIX alberga un nuevo descenso de las temperaturas, que coincide con el momento en el que estudios realizados en otros ámbitos geográficos sitúan el final de las anomalías climáticas relacionadas con la PEH. En los siglos XVII y XVIII los valores térmicos son más suaves, sin detectarse crisis frías de la magnitud de las identificadas en los siglos XVI y XIX. 
Los datos confirman que en la mitad norte de España, al igual que en otros ámbitos geográficos, la característica principal de la evolución de las temperaturas durante la PEH es la presencia de notables crisis frías, de una duración que oscila entre una y tres décadas, en las que los descensos medios rondan los $0,5^{\circ} \mathrm{C}$, sin que se detecte un deterioro térmico continuado a lo largo de los más de tres siglos en los que se extiende este episodio. Sin embargo, el momento en el que se producían las crisis frías más intensas en el área de estudio es en el siglo XVI, frente a lo señalado por las series hemisféricas que presentan al siglo XVII como la centuria más fría dentro de la PEH. A pesar de ello, las principales anomalías frías identificadas, así como los valores más suaves del siglo XVIII coinciden con episodios del mismo signo y cronología descritos en reconstrucciones realizadas en otras zonas de Europa.

De estos resultados se concluye la necesidad de completar un mosaico lo más amplio posible de informaciones paleoclimáticas de carácter regional que permitan identificar en el espacio episodios comunes y comportamientos diferenciales. Ello permitiría interpertar la evolución del clima durante la PEH en términos de Circulación General Atmosférica, y de esta forma comprender mejor las características y trascendencia de este episodio climático, sin duda el más importante del tramo final del Holoceno.

\section{Agradecimientos}

Este trabajo se realizó en el marco del Programa de Grupos de Investigación Consolidados 2003-2004 financiado por el Gobierno de Aragón: grupo S28 "Clima, cambio global y sistemas naturales.

\section{Bibliografía}

Aniol, R.W. (1983). Tree-ring analysis using CATRAS. Dendrochronologia 1, 45-53.

Bauernfeind, W. y Woitek, U. (1999). The influence of climatic change on price flutuations in Germany during the $16^{\text {th }}$ century Price Revolution. Climatic Change, 43, 303-321.
Behringer, W. (1999). Climatic change and Witch-hunting the impact of the Little Ice Age on mentalities. Climatic Change, 43, 335-351.

Briffa, K.R., Bartholin, T.S., Eckstein, D., Jones, P.D., Karlen, W., Schweingruber, F.H., Zetterberg, P. (1990). A 1400-year 
tree-ring record of summer temperatures in Fennoscandia. Nature 346 434-439.

Briffa, K.R., Jones, P.D., Bartholin, T.S., Eckstein, D., Schweingruber, F.H., Karlen, W., Zetterberg, P., Eronen, M. (1992). Fennoscandian summers from A.D. 500 temperature changes on short and long timescales. Climate Dynamics, 7, 111-119.

Briffa, K.R. y Schweingruber, F.H. (1992) Recent dendroclimatic evidence of northern and central European summer temperatures. En Climate Since A.D. 1500, Bradley, R.S. y P.D. Jones (eds.), 366-391. Routledge New York.

Bradley, R.S., Jones, P.D. (1993). Little Ice Age summer temperature variations their nature and relevance to recent global warming trends. The Holocene, 3,367-376.

Briffa, K.R., Jones, P.D., Schweingruber, F.H., Shiyatov, S.G. y Cook, E.R. (1995). Unusual twentieth-century summer warmth in a 1,000-year temperature record from Siberia. Nature 376, 156-159.

Briffa, K.R., Jones, P.D., Schweingruber, F.H., Osborn, T.J. (1998). Influence of volcanic eruptions on Northern Hemisphere summer temperature over the past 600 years, Nature, 393, 350-354

Briffa, K.R., Jones, P.D., Vogel, R.B., Schweingruber, F.H., Baillie, M.G.L. Shiyatov, S.G., Vaganov, E.A. (1999). European Tree-Rings And Climate In The $16^{\text {th }}$ Century. Climatic Change, 43, 151168.

Briffa, K.R., Osborn, T.J., Schweingruber, F.H., Harris, I.C., Jones, P.D., Shiyatov, S.G. y Vaganov, E.A., (2001). Low frequency temperature variations from a northern treering density network. Journal of Geophysical Research 106, 2929-2941.

Briffa, K.R., Osborn, T.J., Schweingruber F.H. (2004). Large-scale temperature inferences from tree rings a review. Global and Planetary Change 40, 11-26

Cook, E.R., Kairiukstis L.A. (1990). Methods of dendrochronology. Kluwer Academic Publishers. Londres, 394 p.

Creus, J. y Puigdefabregas, J. (1983). Climatología histórica y dendrocronología de Pinus nigra Arnold. Avances sobre la Investigación en Bioclimatología. CSIC. 121-127.

Creus, J., Beorlegui, M., Fernández, A. (1995). Reconstrucciones climáticas en Galicia durante las últimas centurias. Estudio Dendrocronológico. Ed. Presidencia Xunta de Galicia. La Coruña. 184 p.

Creus, J., Fernández, A., Manrique E. (1996). Evolución de la temperatura y precipitación anuales desde el año 1400 en el sector central de la depresión del Ebro. Lucas Mallada, 8, 9-27

Creus, J., Saz, M.A. (1999). Estudio de la variabilidad climática del último milenio a partir de series de temperatura y precipitación reconstruidas en el noreste español. La Climatología española en los albores del siglo XXI. In Raso, J.M., Martín Vide J. (eds). Publicaciones de la Asociación Española de Climatología (AEC). Serie A, $1,155-164$.

Crowley, T.J. (2000). Causes of climate change over the past 1000 years, Science, 289270 277.

D'Arrigo, R., Jacoby, G.C. (1992) Dendroclimatic evidence from Northern America. In Climate Since A.D. 1500, Bradley, R.S., P.D. Jones (eds). Routledge New York.

De Vries, J. (1981). Measuring the impact of Climate on History the search for appropriate methodologies. En Climate and History. Studies in interdisciplinary history. (R.I. Rotberg y T.K. Rabb, eds) 
Princeton University Press. Princeton. 1950.

Esper, J., E.R. Cook, Schweingruber F.H. (2002). Low-frequency signals in long treering chronologies for reconstructing past temperature variability, Science, 295, 5563.

Fernández, A, Génova, M, Creus, J, Gutierrez, E. (1996). Dendroclimatological investigation for the last 300-400 years in Central Spain. Radiocarbon, 181-190.

Fernández, A., Manrique, E. (1997). Nueva metodología para la reconstrucción dendroclimática y aplicaciones más importantes. INIA, Madrid, 127 p.

Fritts, H. (1976). Tree rings and climate Academic Press, 567 p.

Fritts, H. (1990). Modelling tree-ring and environmental relationships for dendrochronological analisys. In Forest growth process modelling of responses to environmental stress (Dixon et al, eds). Timers Press. Oregon. 360-382.

Fritts, H. (1991). Reconstructing large-scale climatic patterns from tree-ring data. University of Arizona Press. 420 p.

Galloway, P.R. (1994). Secular changes in the short term preventive, positive and temperature check to population growth in Europe, 1460-1909. Cimatic Change, 26, 363.

Génova, R. (1988). Sincronización entre distintas cronologías del noreste de la Península Ibérica. Options mediterraneénnes, 3 337-340.

Glaser, R. Hagedorn, H. (1991). The climate of Lower Franconia since 1500. Theoretical and Applied Climatology, 43. 101-104.

Glaser, R., Brázdil, R., Pfister, C., Dobrovolny, P., Barriendos, M., Bokwa, A., Camuffo, D., Kotyza, O., Limanowka, D. Racz, L. (1999). Seasonal temperature and precipi- tation fluctuations in selected parts of Europe during the sixteenth century Climatic Change, 43, 169-200.

Goodes, C.M., Palutikof, J.P. y Davies, T.D. (1992). The nature and causes of Climatic Change Assesing the long-term future. Belhaven Press. Londres. 248 pp.

Grove, J.M., Conterio, A. (1995). The climate of Crete in the sixteenth and seventeenth centuries. Climatic Change, 30 223-247.

Guiot, J. (1990). Methods of calibration. In Methods of dendrochronology Cook E.R. Kairiukstis L.A. (eds..), 165-172, Kluwer Academic Publishers.

Guiot, J. (1992). The combination of historical documents and biological data in the reconstruction of climate variations in space and time. Paleoclimate Research, 72, 93-104.

Heino, R., Brázdil, R., Forland, E., Tuomenvirta, H., Alexandersson, H., Benistom, M., Pfister, C., Rebetez, M., Rosenhagen, G., Rösner,S. y Wibig, J. (1999). Progress in study of climatic extremes in Northern and Central Europe. Climatic Change, 42. 151-181.

Holmes, R. (1997). The Dendrochronology Program Library. The International TreeRing Data Bank Program. Laboratory of Tree-Ring Research. University of Arizona. Tucson. 40-74.

Hughes, M.K., Vaganov, E.A., Shiyatov, S., Touchan, R., Funkhouser, G. (1999). Twentieth century summer warmth in northern Yakutia in a 600 year context. The Holocene, 9(5). 629-634.

Jones, P.D., Briffa, K.R., Barnett, T.P., Tett, S.F.B. (1998). High resolution paleoclimatic records for the last millenium interpretation, integration an comparison with General Circulation Model control-run temperatures. The Holocene 8 (4), 455-471. 
Jones, P.D., Briffa, K.R. (2001). The Little Ice Age local and global perspectives. Climatic Change, 48 (1). 5-8.

Kalela-Brundin, M. (1999). Climatic information from tree-rings of Pinus sylvestris L. and a reconstruction of summer temperatures back to AD 1500 in Femundsmarka, eastern Norway, using partial least squares regression (PLS) analysis. The Holocene, 9(1), 59-77.

Koslowski, G., Glaser, R. (1999). Variations in reconstructed ice winter severity in the western Baltic from 1501 to 1995, and their implications for the North Atlantic oscillation. Climatic Change, 41, 175-191

Lamb, H.H. (1977). Climate present, past and future. (Vol II) Mathuen \& Co. Londres.

Lamb, H.H. (1988). Some aspects of the Little Ice Age and other periods of cold, disturbed climate. En Weather, Climate and Human affairs. (H.H. Lamb, ed) Routledge. Londres.

Landsteiner, E. (1999). The crisis of wine production in late sixteenth century Central Europe climatic causes and economic consequences. Climatic Change, 43, 323-334.

Lockwood J.G. (1979). Causes of climate. Edward Arnold. Londres. 260 p.

Luckmann, B.H. (2000). The little Ice Age in the Canadian Rockies. Geomorphology 32, 357-384

Mann, M.E., Bradley R.S. y Hughes, M.K. (1998). Global-scale temperature patterns and climate forcing over the past six centuries. Nature, 392, 779-787.

Mann, M.E. Jones, P.D. (2003). Global surface temperatures over the past two millennia, Geophysical Research Letters,30 (15) 1820

Manrique, E. (1997). Nuevos métodos de reconstrucción dendroclimática. Aplicaciones al estudio climático y fitoclimático del último milenio. Tesis Doctoral, U.P.M, pp 320.

Manrique, E., Fernandez-Cancio, A. (2000). Extreme climatic events in dendroclimatic reconstructions from Spain. Climatic Change, 44 (1-2).123-138.

Nesje, A., Dahl, S.O. (2003). The Little Ice Age, only temperature? The Holocene 13, (1) $39-145$

Pfister, C. (1992). Monthly temperature and precipitation in central Europe 1525-1979. In Climate since $A D$ 1500. Bradley R.S., Jones, P.D. (eds). London, Routledge.

Pfister, C., Brazdil, R. (1999). Climatic variability in sixteenth-century in Europe and its social dimension a synthesis. Climatic Change, 43, 5-53.

Saz, M.A. y Creus, J. (1999). La variabilidad del clima español en el pasado (??) frecuencia de valores extremos de la temperatura y precipitación trimestrales reconstruidas desde el siglo XV. En La Climatología española en los albores del siglo XXI. (Raso, J.M. y Martín Vide J. eds). Publicaciones de la Asociación Española de Climatología (AEC). Serie A, no 1, 501510 .

Saz, M.A. y Creus, J. (2000). Comportamiento cíclico de la precipitación estival en el Noreste de España. A Geofísica e a Geodesia no século XXI. Instituto Geofísico do Infante D. Luís (IGIDL). Lisboa. 515516.

Saz, M.A. y Creus, J. (2001a). El clima del Pirineo Centro-Oriental desde el siglo $\mathrm{XV}(? ?)$ estudio dendroclimático del observatorio de Capdella. Boletín Glaciológico Aragonés, 2, 37-80.

Saz, M.A. y Creus, J. (2001b). El clima de La Rioja desde el siglo XV. Reconstrucciones dendroclimáticas del observatorio de Haro. Zubia, 13, 41-64. 
Saz, M.A. (2003). Temperaturas y precipitaciones en la mitad norte de España desde el siglo XV. Estudio dendroclimático. Consejo de Protección de la Naturaleza. Diputación General de Aragón. pp 294.

Saz, M.A. Creus, J., Cuadrat, J.M. (2003). Mean summer temperatures dendroclimatic reconstruction in Northeast of Spain. Comparison with other regional studies. Geophysical Research Abstracts, vol 5.

Saz, MA. y Creus, J. (2003). Thermische anomalien im nördlichen Spanien während des 16. Jahrhunderts erste zeichen der kleinen eiszeit. In Landnutzungswandel und Landdegradation in Spanien. Marzolff, I., Ries, J.B., De La Riva, J. Seeger, M. (eds). 121-141.

Saz, M.A. y Creus, J. (2004). Evolución de las temperaturas en Villacarriedo (Cantabria) durante la Pequeña Edad del Hielo (PEH). En García Cordón et al (eds). El clima entre el mar y la montaña. Universidad de Cantabria. pp 407-418

Saz, M.A., Creus, J., Cuadrat, J.M. (2004). La Pequeña Edad del Hielo en Galicia reconstrucción de las temperaturas de Labacolla entre los siglos XVI y XIX. Xeográfica 4. 141-161
Saz, M.A. y Creus, J. (2005). Las precipitaciones durante la época cálida en el sur de la provincia de Alicante desde 1550 a 1915. Revista de Historia Moderna, 23. 35-48

Saz, MA (2005). La Pequeña Edad del Hielo en el Noreste Español. Reconstrucción dendroclimática de las temperaturas medias de invierno y verano. Boletín Glaciológico Aragonés, 6, 9-36

Serre-Bachet, F. (1994). Annual and summer mean temperature reconstructions from tree rings in western and southern Europe since A.D. 1500 with special reference to the late Maunder Minimum. In Climatic Trends and Anomalies in Europe 16751715 Frenzel, B. (ed). 265-274. European Science Foundation, Strasbourg.

Tarussov, A. (1992). The artic from Svalbard to Severnaya Zemlya climatic reconstructions from ice cores. In Climate since A.D. 1500. Bradley R.S. Jones P.D. (eds) London. Routledge

Villa, D; Guerra, J, Corres, R, (1985). Análisis estadístico de la pluviometría de la península Ibérica. Instituto Nacional de Meteorología, Serie A-132, pp 17. 